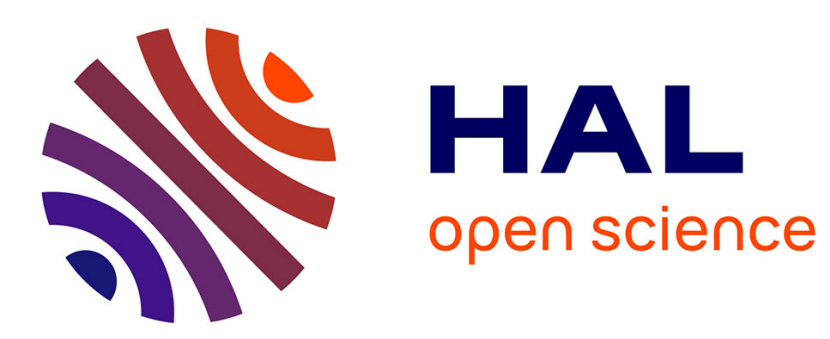

\title{
La prudence du prince: les assassinats ciblés et leurs conséquences stratégiques, internationales et politiques
} Amelie Ferey

\section{To cite this version:}

Amelie Ferey. La prudence du prince: les assassinats ciblés et leurs conséquences stratégiques, internationales et politiques. Les Champs de Mars: revue d'études sur la guerre et la paix, 2018. hal-01802560

\section{HAL Id: hal-01802560 \\ https://univ-lyon3.hal.science/hal-01802560}

Submitted on 29 May 2018

HAL is a multi-disciplinary open access archive for the deposit and dissemination of scientific research documents, whether they are published or not. The documents may come from teaching and research institutions in France or abroad, or from public or private research centers.
L'archive ouverte pluridisciplinaire HAL, est destinée au dépôt et à la diffusion de documents scientifiques de niveau recherche, publiés ou non, émanant des établissements d'enseignement et de recherche français ou étrangers, des laboratoires publics ou privés. 


\title{
LA PRUDENCE DU PRINCE : LES ASSASSINATS CIBLÉS ET LEURS CONSÉQUENCES STRATÉGIQUES, INTERNATIONALES ET POLITIQUES
}

\author{
Amélie Ferey
}

Presses de Sciences Po (P.F.N.S.P.)| «Les Champs de Mars »

2018/1 N³0 + Supplément | pages 467 à 478

ISSN 1253-1871

ISBN 9782724635423

Article disponible en ligne à l'adresse :

https://www.cairn.info/revue-les-champs-de-mars-2018-1-page-467.htm

\section{Pour citer cet article :}

Amélie Ferey, « La prudence du prince : les assassinats ciblés et leurs conséquences stratégiques, internationales et politiques », Les Champs de Mars 2018/1 (Nº $30+$ Supplément), p. 467-478.

Distribution électronique Cairn.info pour Presses de Sciences Po (P.F.N.S.P.).

(C) Presses de Sciences Po (P.F.N.S.P.). Tous droits réservés pour tous pays.

La reproduction ou représentation de cet article, notamment par photocopie, n'est autorisée que dans les limites des conditions générales d'utilisation du site ou, le cas échéant, des conditions générales de la licence souscrite par votre établissement. Toute autre reproduction ou représentation, en tout ou partie, sous quelque forme et de quelque manière que ce soit, est interdite sauf accord préalable et écrit de l'éditeur, en dehors des cas prévus par la législation en vigueur en France. Il est précisé que son stockage dans une base de données est également interdit. 


\title{
LA PRUDENCE DU PRINCE : \\ LES ASSASSINATS CIBLÉS \\ ET LEURS CONSÉQUENCES STRATÉGIQUES, INTERNATIONALES ET POLITIQUES
}

\author{
AMÉLIE FEREY
}

Résumé : Les assassinats ciblés désignent l'utilisation de la force létale par un sujet de droit international avec l'intention préméditée et délibérée de tuer individuellement les personnes sélectionnées qui ne se trouvent pas sous la garde des États concernés. L'objet de cet article est l'analyse de cette critique dite "conséquentialiste" des assassinats ciblés qui examine leurs résultats. Ces opérations sont-elles contre-productives? Incarnent-elles une utilisation inconséquente de la force ? Je distingue trois ordres de conséquences : stratégiques - produisent-elles des résultats efficaces?; internationales - quelles sont leurs conséquences pour le principe de souveraineté ?; et politiques - quel est leur impact sur le caractère démocratique des États qui les emploient? Fragilisent-elles l'État de droit? Je conclus mon étude par un examen de la situation française qui fait figure d'exception parmi les pays cités. Il existe une incertitude de l'État français sur la position à adopter vis-à-vis de cet emploi de la force, qui complique la délibération collective sur sa légitimité.

\begin{abstract}
Targeted killings refer to the use of lethal force by a subject of international law with the premeditated and deliberate intent to kill individually selected persons who are not in the custody of those responsible for targeting. In this paper, I discuss what I call the "consequentialist critique" of targeted killings, analysing their results. Are these operations counter-productive? Do they constitute a reckless use of force? We distinguish between three types of consequences: strategic (are they effective?), international (do they weaken the principle of sovereignty?), and political (are they a threat to the rule of Law in the States that resort to them?). I conclude by focusing on the position of France within this debate. France is an exception among other countries in that there is an ambiguity on its public discourse on targeted killings, which complicates the collective discussion on their legitimacy.
\end{abstract}

\section{INTRODUCTION}

Les assassinats ciblés désignent l'utilisation de la force létale par un sujet de droit international avec l'intention préméditée et délibérée de tuer individuellement les personnes sélectionnées qui ne se 
trouvent pas sous la garde des États concernés ${ }^{1}$. Ils font partie de l'arsenal contre-terroriste dont se sont dotés plusieurs pays. Les États-Unis, Israël, la Russie, le Royaume-Uni, le Pakistan et la France reconnaissent publiquement ou semi-publiquement y avoir recours. Ces opérations ont lieu aussi bien dans des théâtres d'opération "actifs» qu'en dehors des zones de guerre déclarées, comme au Yémen, au Pakistan ou en Somalie. Leur légalité varie en fonction des endroits dans lesquels elles sont conduites. Visant à éliminer l'ennemi avant qu'il ne nuise, cette tactique est à distinguer des moyens à l'aide desquels elle est effectuée, qui varient : commandos, explosifs, missiles tirés depuis des avions de chasse ou des hélicoptères. L'utilisation récente de drones armés a déclenché une forte controverse qui doit être différenciée de l'assassinat ciblé en tant que tel.

Ces pratiques sont réputées connaître peu d'opposition de principe - les journaux couvrant les assassinats ciblés relaient régulièrement des sondages affirmant que la majorité des citoyens sont en leur faveur ${ }^{2}$. Les interrogations suscitées par ceux-ci seraient davantage d'ordre pragmatique : ces opérations sont-elles contre-productives? Incarnent-elles une utilisation inconséquente de la force? L'objet de cet article est l'analyse de cette critique dite " conséquentialiste » des assassinats ciblés qui examine leurs résultats. Je distingue trois ordres de conséquences : stratégiques - produisent-elles des résultats efficaces?; internationales - quelles sont leurs conséquences pour le principe de souveraineté ?; et politiques - quel est leur impact sur le caractère démocratique des États qui les emploient? Fragilisent-elles l'État de droit? Je conclus mon étude par un examen de la situation française qui fait figure d'exception parmi les pays cités. Il existe une incertitude de l'État français sur la position à adopter vis-à-vis de cet emploi de la force qui complique la délibération collective sur sa légitimité.

\footnotetext{
1. La dénomination de ces opérations fait débat. Le terme anglais targeted killing peut se traduire par "élimination ciblée " ou par "assassinat ciblé ". Le terme hébreu sikud memukad signifie littéralement "prévention ciblée ", ce qui oblitère la dimension létale. Nous parlons ici d'assassinat ciblé afin de souligner le caractère personnalisé et prémédité de cette pratique ainsi que son ambiguité légale, bien que cette expression soit un pléonasme.

2. Le sondage AP-GFK concluait en 2015 que $60 \%$ des Américains étaient en faveur de la politique de frappes par drones.
} 


\section{LES CONSÉQUENCES STRATÉGIQUES : LES ASSASSINATS CIBLÉS SONT-ILS CONTRE-PRODUCTIFS ?}

L'espoir d'une réponse définitive à la question de l'opportunité stratégique des assassinats ciblés doit être abandonné. Nombre d'études quantitatives caressant le but d'analyser scientifiquement leur efficacité et de trancher cette question ont été publiées ${ }^{3}$. Elles me semblent peu concluantes car elles se heurtent à au moins cinq limitations épistémologiques. Premièrement, elles sont fragilisées par un accès partiel aux informations. De nombreuses données cruciales, telles que le nombre de frappes ayant lieu par an, leur létalité, le taux d'erreur et le taux d'abandon des missions, sont confidentielles, ce qui limite la crédibilité d'une recherche indépendante. Ensuite, la validité même des études quantitatives est sujette à caution. Si des corrélations peuvent être établies entre différents indicateurs - le nombre d'assassinats ciblés, la fréquence et la létalité des attaques terroristes -, en déduire une causalité me semble périlleux. Comment isoler la variable "assassinats ciblés » du grand nombre de facteurs connexes, tels que le contexte politique, la perspective éventuelle de négociations, les dynamiques politiques internes à l'organisation visée ou le contexte international? Quelles sont les variables de contrôle pouvant être utilisées ? Un tel passage de la corrélation à la causalité est donc compliqué par la contre-factualité qu'il suppose. De surcroît, la portée comparative de ces études est à relativiser. L'établissement de tendances statistiques est à distinguer de celui de lois générales. Si les études sur les assassinats ciblés israéliens et américains peuvent fournir des éléments de mise en perspective de l'opportunité pour la France d'adopter une telle stratégie, elles ne constituent en rien une garantie de réussite. L'environnement stratégique et l'intérêt national de ces pays ne sont pas équivalents. Enfin, les catégories analytiques sur lesquelles reposent ces enquêtes me paraissent douteuses. Elles expliquent la vulnérabilité d'une organisation terroriste par son agenda politique défini par des revendications nationalistes ou religieuses et par ses caractéristiques organisationnelles - sa durée de vie, son caractère plus ou moins

\footnotetext{
3. Voir Mohammed M. Hafez, Joseph M. Hatfield, "Do targeted assassinations work? A multivariate analysis of Israel's controversial tactic during Al-Aqsa uprising", Studies in Conflict \& Terrorism, 29 , 2006 ; Patrick B. Johnston, «Does decapitation work? Assessing the effectiveness of leadership targeting in counterinsurgency campaigns ", International Security, 36, 2012 ; Brian C. Pryce, "Targeting top terrorists. How leadership decapitation contributes to counterterrorism ", International Security, 36 (4), 2012, p. 9-46.
} 
personnalisé. Or, ces catégories ne correspondent pas toujours aux réalités empiriques d'organisations dont la spécificité et les capacités d'adaptation doivent être prises au sérieux.

In fine, l'échec des études quantitatives à déterminer avec certitude l'efficacité des assassinats ciblés témoigne de leur méconnaissance de la nature politique des faits étudiés. Il n'existe ainsi pas d'évaluation définitive sur laquelle une défense conséquentialiste des assassinats ciblés puisse s'appuyer. Toutefois, reconnaître l'inefficacité de l'approche quantitative dans l'analyse de la stratégie militaire ne condamne pas au silence. Les assassinats ciblés répondent à certains objectifs tactiques d'identification de l'ennemi, de nuisance opérationnelle et de dissuasion. Leur succès tactique est souvent masqué par une indétermination des buts stratégiques auxquels ils répondent.

\section{Un certain succès tactique}

Les assassinats ciblés visent des buts tactiques, au premier rang desquels se trouve l'identification de l'ennemi. Pour Michael L. Gross, ces "tueries nommées " (named killing) seraient un moyen de permettre aux soldats de reconnaître les combattants ennemis malgré leur absence de signes distinctifs. Elles assureraient un meilleur respect du principe de distinction ${ }^{4}$. Cet impératif tactique de «ciblage » de l'ennemi renvoie à une difficulté d'ordre technique : comment déterminer correctement l'identité des personnes à tenir en ligne de mire? Cela requiert une très bonne connaissance des réseaux visés. Les agences de renseignement sont ainsi naturellement sollicitées par l'armée. À cet égard, la pratique américaine des signatures strikes, où l'identification des cibles est faite de manière peu précise, sur la seule base de l'âge et de la localisation des personnes qui porteraient alors la "signature " des militants terroristes, a été très critiquée, au point que l'administration Obama affirme l'avoir abandonnée en $2013^{5}$. Un autre problème est celui du calibrage adéquat du spectre des cibles visées. Faut-il viser les personnes occupant des responsabilités secondaires dans l'organisation, les mid levels, ou s'en tenir aux personnalités les plus haut

4. Michael L. Gross, Moral Dilemmas of Modern War. Torture, Assassination, and Blackmail in an Age of Asymmetric Conflict, Cambridge, Cambridge University Press, 2010, p. 103.

5. Dan de Luce, Paul Mclearly, "Obama's most dangerous drone tactic is here to stay", Foreign Policy, 5 avril 2016 . 
placées, les high value targets ${ }^{6}$ ? La distinction entre les branches armée et politique incite-t-elle les organisations à dissocier les deux activités ${ }^{7}$ ? La perspective d'un mouvement d'élargissement continu des cibles effraye les responsables militaires et politiques. Une politique efficace d'assassinats ciblés demande également une mesure précise de l'opportunité stratégique de tuer les cibles visées. Cela requiert une très bonne connaissance des compétences et de l'organisation interne de ces dernières afin de savoir qui sera éventuellement leurs remplaçants. Le cas classique d'une erreur stratégique est la frappe israélienne en 1992 contre le leader du Hezbollah Abbas Al-Moussawi qui fut remplacé par Hassan Nasrallah, plus radical que son prédécesseur. Enfin, il est impératif de s'assurer de la fiabilité des informations sur la base desquelles sont réalisées les frappes. La manipulation des programmes par les alliés à des fins de répression politique est une possibilité qui ne peut être absolument écartée. L'argument a été utilisé dans le cas du Vietnam, du Pakistan et du Yémen.

Leur deuxième impératif tactique est la nuisance opérationnelle. Les assassinats ciblés auraient un effet direct sur les organisations visées : ils sèmeraient la confusion, compliqueraient les communications en obligeant les membres à se cacher et en leur ôtant des ressources humaines cruciales. Cette forte capacité de nuisance se traduirait par une diminution des attaques ${ }^{8}$.

Troisièmement, ils répondraient à un objectif indirect en érodant la détermination à combattre. Pour l'ancien directeur du Shin Beth Avi Dichter, les assassinats ciblés participent d'une stratégie de dissuasion visant à affecter le "sens de la sécurité » des organisations armées ${ }^{9}$. Comme l'étaient les stratégies d'attrition, la capacité des assassinats ciblés à dissuader les organisations visées est sujette à

\footnotetext{
6. Matt Frantel, «The ABCs of HVT. Key lessons from high value targeting campaigns against insurgents and terrorists", Studies in Conflict \& Terrorism, 34 (1), 2010.

7. Cette stratégie a été mise en place en Israël. Il existe toutefois des contre-exemples : Israël s'est parfois autorisé à tuer des «idéologues », comme Khaled Meshal, le Dr. Thabet Thabet, le cheick Yassine. Ce serait également le cas des États-Unis avec la frappe ciblant Anouar al-Aulaqi.

8. Oussama Ben Laden s'en serait lui-même inquiété, comme l'indiquent les documents saisis à son domicile lors de l'opération "Trident de Neptune ». John O. Brennan le cite dans son discours d'avril 2012 "Par exemple, Ben Laden s'est inquiété de - et je cite - "l'ascension de leaders bas placés qui ne sont pas aussi expérimentés et qui mènera à répéter des erreurs". "

9. Moshe Yaalon, Avi Dichter, Dennis Ross, "Lessons from the fight against terrorism ", conférence donnée au Washington Institute, retranscription, le 29 décembre 2005.
} 
caution ${ }^{10}$. Un désir de vengeance poussant aux représailles pourrait au contraire augmenter la possibilité d'attaques. Le colonel de l'armée de défense israélienne Pnina Baruch Sharvit en est bien consciente: pour elle, "faire des assassinats ciblés revient à jeter une allumette $^{11}$ ". Ils contribueraient à renforcer le soutien dont le groupe visé dispose au sein de la population et créeraient des martyrs facilement exploitables. À titre d'exemple, les vidéos de l'imam Anouar Al Aulaqi ont ainsi été davantage visionnées après son élimination par un drone américain ${ }^{12}$.

\section{Quelle finalité stratégique?}

L'évaluation de l'efficacité stratégique des assassinats ciblés se heurte principalement à l'incertitude sur leur finalité. Ont-ils pour objectif d'éradiquer les insurrections et d'annihiler leurs cellules terroristes? De dissuader de nouveaux recrutements? De réduire le nombre d'attaques sur le sol national ? La littérature sur ces derniers fait émerger une pluralité de buts parfois contradictoires. Un premier élément de réponse se trouve dans la distinction entre stratégie et tactique. Elle permet de comprendre que les assassinats ciblés ne peuvent être à eux seuls le "remède miracle » éradiquant les menaces. C'est un reproche qui leur est pourtant régulièrement adressé : depuis leur mise en place après le 11 septembre 2001, le monde n'aurait pas connu le décroît de terrorisme qu'ils promettaient. Or les assassinats ciblés ne constituent pas une stratégie d'ensemble contre les acteurs irréguliers, bien qu'ils soient parfois présentés comme tels ${ }^{13}$. $\mathrm{Au}$ contraire, ils relèvent davantage d'une tactique qui doit être combinée à d'autres en fonction du contexte d'emploi.

\footnotetext{
10. Robert Pape, Bombing to Win. Air Power and Coercion in War, Ithaca (N. Y.), Cornell University Press, 1996. Avi Dichter, qui avait pourtant pris ses distances avec les assassinats ciblés en 2006, renoue avec cette tactique en la liant clairement à une stratégie d'attrition visant à cibler les infrastructures civiles dans la bande de Gaza. Celui-ci déclare qu'Israël devrait «frapper les membres clés de l'infrastructure de la terreur à Gaza et en même temps frapper les infrastructures civiles dans la bande ». Le ministre Uri Landau déclare quant à lui que les leaders du Hamas « devraient aller dans des bunkers et s'inquiéter de leur bonne santé personnelle continuellement». Voir Gil Ronen, "Min. Landau, MK Dichter. Renew targeted killings of terrorists ", 24 mars 2011.

11. Colonel Pnina Baruch-Sharvit, entretien avec l'auteure.

12. A. J. Chavar, Scott Shane, "Dead, but not forgotten", New York Times, 27 août 2015.

13. Herb Keinon, "Yadlin. Targeted assassinations important, but not a "silver bullet" ", The Jerusalem Post, 21 août 2014.
} 
Cette confusion entre tactique et stratégie est régulièrement dénoncée par les acteurs mettant en place ces politiques ${ }^{14}$. Elle témoigne du conflit entre deux rationalités. La première, politique, vise à formuler des solutions claires susceptibles de rassurer les opinions publiques. Les assassinats ciblés permettraient de faire l'économie d'une stratégie d'ensemble plus ambitieuse mais aussi plus coûteuse politiquement et économiquement. À l'opposé, la rationalité militaire, à l'abri de ces contraintes politiques, est souvent considérée comme une variable d'ajustement de ces dernières. La stratégie serait alors sacrifiée sur l'autel de la politique interne.

En outre, s'il y a persistance des attaques terroristes dans les sociétés pratiquant les assassinats ciblés, leurs modalités ont muté. Les études notent une baisse des attaques à la bombe, contrecarrée par une augmentation du terrorisme sur le mode du «loup solitaire » : des individus sans lien apparent avec des organisations terroristes agissent de leur propre initiative depuis le territoire des pays visés ${ }^{15}$. Leurs actions sont particulièrement difficiles à endiguer pour les pouvoirs publics. Le succès relatif des assassinats ciblés ne conduirait pas à un arrêt du terrorisme mais à sa mutation dans une forme moins létale. Les États-Unis n'ont ainsi pas subi d'attaques de l'ampleur de celle du 11 septembre 2001 et, en Israël, la létalité de l'Intifada dite des "couteaux » est sans commune mesure avec celle de la seconde Intifada ${ }^{16}$.

Pour résumer, les assassinats ciblés comportent des avantages tactiques mais ils ne constituent pas une stratégie d'ensemble visant à lutter contre les menaces asymétriques. Ces derniers expriment une préférence politique qui indique un rapport averse au risque et une

\footnotetext{
14. Dans le documentaire The Gatekeepers, les directeurs du Shin Beth racontent l'instrumentalisation des assassinats ciblés par les politiques dans la perspective de réduire les coûts et de formuler une "solution " simple au terrorisme dont les opinions publiques sont friandes. Droh Moreh, The Gatekeepers, 2012.

15. L'expression aurait été inventée par les suprématistes blancs américains Alex Curtis et Tom Metzger dans les années 1990. L'attentat de Nice commis par Mohamed Lahouaiej Bouhlel le 14 juillet 2016 en est un exemple. Toutefois, le terme est à prendre avec précaution en raison de la difficulté à établir avec certitude l'absence de liens avec des organisations établies telles que ISIS. Voir Daveed Gartenstein-Ross, Nathaniel Barr, "The myth of lone wolf terrorism ", Foreign Policy, 26 juillet 2016.

16. Selon les chiffres de l'organisation non gouvernementale israélienne Bt'selem, en avril 2008, 1053 Israéliens ont trouvé la mort dans des attaques liées à la seconde Intifada et 4775 Palestiniens ont été tués par les forces israéliennes. Par comparaison, d’après les chiffres donnés par le ministère des Affaires étrangères israélien, de septembre 2015 à mars 2017, soit la période couvrant l'Intifada dite des couteaux, 47 Israéliens ont trouvé la mort.
} 
volonté de préserver la vie des soldats en évitant un déploiement terrestre de plus grande ampleur. La légitimité de leur recours ne peut donc pas faire l'économie d'une argumentation politique. Les assassinats ciblés sont une solution de court terme mais, s'ils ne sont pas accompagnés par une réflexion plus large, ils sont condamnés à être un coup d'épée dans l'eau.

\section{LES CONSÉQUENCES À L'INTERNATIONAL : LES ASSASSINATS CIBLÉS PARTICIPENT-ILS AU DÉSORDRE MONDIAL ?}

Les assassinats ciblés sont suspectés de constituer un permis de tuer à l'échelle mondiale, sapant les bases du principe d'intégrité territoriale. Cette peur est ancrée dans la technologie des drones armés qui offre la possibilité de pénétrer des espaces aériens et de projeter sa force. Faisant fi des frontières, ils permettraient aux "puissants » de tuer comme bon leur semble, au mépris des règles du droit international et sans considération pour la souveraineté des États. Ils porteraient donc le germe d'une instabilité mondiale, transformant de facto le monde en champ de bataille, en autorisant une ingérence intolérable.

Dix ans après les premiers assassinats ciblés conduits par Israël et les États-Unis, le monde ne s'est clairement pas transformé en un Far-West grandeur nature. Les chefs d'État sont tout aussi protégés qu'ils l'étaient avant les premières opérations. Il faut à cet égard souligner que la norme interdisant le recours à l'assassinat n'a jamais concerné les acteurs non étatiques : ceux-ci ne disposent pas de la protection accordée aux souverains et sont donc conçus comme des cibles légitimes ${ }^{17}$. Face au désordre imposé par l'émergence de nouveaux acteurs non étatiques, l'assassinat ciblé fonctionnerait plutôt comme un renforcement de la souveraineté étatique. Il permettrait de réaffirmer l'autorité de l'État susceptible de tenir à distance les velléités de pouvoir de groupes armés.

S'il est clair que les assassinats ciblés renforcent la puissance des États qui les emploient, ils peuvent également contribuer à affirmer la souveraineté des États plus vulnérables. L'exemple du Pakistan

\footnotetext{
17. Hugo Grotius précise sa réflexion sur les acteurs illégitimes dans un ouvrage antérieur, Mare Liberum, écrit en 1609. Les assassinats ciblés ne concernent en effet pas des dirigeants souverains : il n'y a pas eu de tentative connues de frappe ciblée contre al-Assad par exemple, malgré la menace qu'il fait peser sur le système international.
} 
reflète la manière dont ils peuvent être utilisés pour asseoir indirectement la souveraineté territoriale des États en proie à des groupes contestant leur autorité. Les États-Unis ont conduit des frappes de drones dans cette région depuis 2004, avec une intensification de ces dernières en 2013 sous la présidence Obama. Hautement impopulaires, elles sont dénoncées par le président Nawaz Sharif en des termes très clairs dans un discours prononcé en 2013 sur le sol américain :

L'utilisation des drones n'est pas seulement une violation continue de notre intégrité territoriale mais est aussi désastreuse pour notre résolution et nos efforts pour éliminer le terrorisme dans notre pays ${ }^{18}$.

La Haute Cour de Peshawar les condamne au nom du principe de non-ingérence. Toutefois, les révélations de Wikileaks en 2010 montrent que les frappes de drones américaines ont été, au moins jusqu'à cette date, soutenues et parfois encouragées par le pouvoir central ${ }^{19}$. En avril 2013, dans un revirement spectaculaire, l'ancien président pakistanais Pervez Musharraf reconnaît, au cours d'une interview accordée à $\mathrm{CNN}$, que son gouvernement a secrètement donné son accord à la conduite de frappes américaines sur son territoire. Il restreint toutefois la portée de son propos «à quelques occasions seulement, lorsque la cible est absolument isolée et sans risque de dommage collatéral ${ }^{20}$ ». Comment comprendre cette schizophrénie de l'État pakistanais? Comme l'explique Akbar Ahmed, ancien ambassadeur de ce pays aux États-Unis, les frappes ont principalement lieu dans les zones tribales situées à la frontière entre le Pakistan et l'Afghanistan. Elles abritent des groupes qui contestent fortement la légitimité du gouvernement central. Akbar Ahmed propose ainsi une grille de lecture alternative à la guerre contre le terrorisme : pour lui, il s'agit plutôt d'un conflit entre un islam tribal et un pouvoir étatique central. Les frappes de drones participeraient donc au renforcement de la souveraineté du gouvernement

\footnotetext{
18. "Pakistani PM urges US to stop drone strikes ", Voice of America, 22 octobre 2013.

19. Tim Lister, "WikiLeaks. Pakistan quietly approved drone attacks, US special units », CNN, 2 décembre 2010. Dans un câble diplomatique, l'ambassadrice américaine au Pakistan rapporte que le Premier ministre pakistanais d'alors, Yousaf Raza Gilan (Parti du peuple pakistanais) aurait déclaré : "I don't care if they do it as long as they get the right people. We'll protest in the National Assembly and then ignore il " ("Cela ne me dérange pas qu'ils le fassent pourvu qu'ils s'en prennent aux bonnes personnes. Nous protesterons à l'Assemblée nationale puis nous l'ignorerons ").

20. Greg Miller, "Yemeni president acknowledges approving US drone strike », CNN, 2 décembre 2010.
} 
pakistanais contre les tribus et les groupes armés la contestant. L'enjeu politique pour le gouvernement pakistanais ne serait pas la violation de sa souveraineté mais la place donnée à ses services de renseignement et à l'armée pakistanaise dans la coopération avec l'allié américain pour conduire les frappes ${ }^{21}$.

\section{LES CONSÉQUENCES SUR LES INSTITUTIONS DÉMOCRATIQUES : VERS LA FORMULATION D'UNE EXIGENCE DE TRANSPARENCE VIS-À-VIS DES FORCES ARMÉES ?}

Le dernier ordre de conséquences potentiellement négatives des assassinats ciblés interroge leur impact sur la nature des États l'utilisant. Sous quelles conditions l'État peut-il légitimement ôter la vie d'un individu? Le champ de questionnement ouvert est très vaste et le format de l'article interdit toute velléité d'exhaustivité sur ce sujet. Je m'en tiendrai ici à l'analyse des stratégies de médiatisation. Si les États ont souvent pris la liberté de tuer leur ennemi à l'ombre des services secrets ${ }^{22}$, la spécificité des assassinats ciblés réside justement dans leur publicité. Plusieurs raisons peuvent l'expliquer : la volonté de rassurer une population face à la montée de la menace terroriste, la possible compatibilité de cette tactique avec les normes du droit international, son aspect dissuasif. Mais surtout, les États seraient plus enclins à utiliser des technologies militaires pour ne pas risquer la vie de leurs citoyens, au besoin en dehors de zones de guerre. Lancer un missile à l'aide d'un drone est plus aisé qu'envoyer un agent secret déguisé en civil, même si cela entraîne une nécessaire reconnaissance publique de l'utilisation de la force. Dans un monde de l'information, il est malaisé de conduire des opérations «secrètes ». En prenant les devants, les États conservent leur avantage dans la bataille de la légitimité, décisive dans la lutte qui les oppose aux acteurs non étatiques.

Deux stratégies de publicisation se dégagent : l'une d'ouverture; l'autre de fermeture. Israël incarne la première : l'État hébreu a choisi de privilégier la reconnaissance de ces pratiques et met en avant leur légalité. La Cour suprême israélienne, saisie sur ce dossier, se déclare compétente et légalise sous conditions les assassinats ciblés en 2006.

\footnotetext{
21. Akbar S. Ahmed, The Thistle and the Drone. How America's War on Terror Became a Global War on Tribal Islam, Washington (Wash.), Brookings Institution, 2013.

22. Sur le cas français, voir à ce propos le livre très instructif de Vincent Nouzille, Les Tueurs de la République, Paris, Fayard, 2015.
} 
Les motivations de cette stratégie de légalisation ne sont pas seulement l'expression d'un amour pour la légalité internationale. Son enjeu est le contournement des actions en justice intentées contre Israël. Suite à l'opération ayant tué Salah Shehadeh en 2002, une cour espagnole entame l'instruction d'un procès pour crime de guerre par la compétence universelle. Au rang des accusés figuraient notamment le chef d'état-major et le ministre de la Défense israéliens. Les charges ont été abandonnées après la reprise en main juridique de l'affaire par les instances de justice israéliennes. Ainsi, imposer un cadre légal national en concédant une forme de transparence sur les pratiques peut permettre de se prémunir d'éventuelles poursuites par des instances extra-nationales. Cela a pour deuxième avantage de clore le débat : les assassinats ciblés sont aujourd'hui acceptés par les différentes composantes de la politique israélienne.

La stratégie de médiatisation choisie par les États-Unis est au contraire marquée par l'opacité. Les informations disponibles sur les programmes américains sont majoritairement issues de sources non officielles. Les journalistes de The Intercept qui avaient révélé en 2015, sous le titre de "Drone papers", des informations données par des pilotes de drone devenus lanceurs d'alerte mènent une bataille féroce au nom de la liberté de la presse pour obtenir une levée partielle du secret défense. Cet agenda politique est partagé par les parlementaires américains. Une proposition de loi intitulée Targeted Lethal Force Transparency Act avait été déposée au Congrès dès 2013 afin d'y remédier, sans succès. Le caractère discrétionnaire du pouvoir exécutif sur ces questions est fortement défendu : pour l'administration Obama comme pour celle de Donald Trump, il n'est pas question de faire contrôler cette politique par les cours de justice. Le cas du binational Anouar Al-Aulaqi, un prédicateur lié à Al-Qaeda tué au Yémen en 2011, l'illustre bien. Les cours américaines chargées par le père d'Anouar Al-Aulaqi assisté de l'ACLU (American Civil Liberties Union) de contrôler la légalité de cette frappe se sont déclarées non compétentes en vertu de la séparation des pouvoirs.

Les conséquences de ce choix de médiatisation pour la légitimité des programmes sont importantes. L'hypothèse selon laquelle les stratégies d'ouverture conduisent à clore le débat alors que celles maintenant le flou sur ces activités incitent à une critique et à des confusions tend à s'imposer. 


\section{CONCLUSION : UN RETARD FRANÇAIS ?}

$\mathrm{Si}$, en Israël et aux États-Unis, les interventions armées sont davantage soumises à la nécessité d'être justifiées publiquement, la France fait figure d'exception. Elle dénote par l'absence d'une doctrine d'emploi claire et d'un discours officiel d'explication de ces pratiques. Cette communication parfois brouillonne suscite des interrogations et conduit à des contresens sur la finalité et l'ampleur des frappes conduites. La polémique suscitée par les "révélations » de François Hollande sur la conduite d'assassinats ciblés en 2016 peut surprendre. De part et d'autre du spectre politique, les condamnations sont très vives, alors que l'on aurait pu s'attendre à voir la société civile française se réjouir du progrès de son contrôle sur les actions prises en son nom par le pouvoir exécutif ${ }^{23}$. Elle pose la question suivante: que faut-il dire au public dans le contexte de la guerre contre le terrorisme ${ }^{24}$ ? Cette controverse masque un consensus politique sur le recours à cette pratique. En se concentrant sur la forme, elle oblitère des questions plus épineuses : pourquoi choisir l'élimination plutôt que la capture? Quels sont les risques que nous sommes prêts à faire peser sur nos soldats pour traduire les djihadistes en justice? Sommes-nous prêts à conduire des frappes contre des djihadistes français? Et enfin, quelle reformulation des normes juridiques enserrant l'usage de la force voulons-nous conduire?

Ces questions sont décisives pour notre démocratie et méritent d'être débattues publiquement. Le récent rapport du Sénat sur cette question déplore l'absence de ces débats en France en même temps qu'il affirme la nécessité stratégique d'armer les drones. Une clarification sous la forme d'une doctrine d'emploi serait à cet égard la bienvenue.

23. Les candidats de la Belle Alliance populaire ont presque tous condamné la divulgation de ces "secrets d'État ", bien quaucun n'ait émis de désaccord de fond sur le recours à cette pratique. Le député LR Eric Ciotti a intenté une procédure auprès du procureur de la République pour violation du secret défense. 24. Cela est d'autant plus surprenant que le livre de Gérard Davet et Fabrice Lhomme ne nous apprend pas l'existence du programme d’assassinats ciblés mais précise ses procédures. Manuel Valls l'avait reconnu lors d'un déplacement en Jordanie à l'automne 2015. 